\title{
THz Beam Shaper Realizing Fan-Out Patterns
}

\author{
K. Liebert ${ }^{1}$ - M. Rachon ${ }^{1} \cdot$ A. Siemion ${ }^{1} \cdot$ J. Suszek $^{1}$ • \\ D. But ${ }^{2} \cdot$ W. Knap ${ }^{2} \cdot$ M. Sypek ${ }^{1}$
}

Received: 16 February 2017 / Accepted: 11 May 2017 /

Published online: 31 May 2017

(C) The Author(s) 2017. This article is an open access publication

\begin{abstract}
Fan-out elements create an array of beams radiating at particular angles along the propagation axis. Therefore, they are able to form a matrix of equidistant spots in the far-field diffraction region. In this work, we report on the first fan-out structures designed for the $\mathrm{THz}$ range of radiation. Two types of light-dividing fan-out structures are demonstrated: (i) the $3 \times 1$ matrix fan-out structure based on the optimized binary phase grating and (ii) the $3 \times 3$ fan-out structure designed on the basis of the well-known Dammann grating. The structures were generated numerically and manufactured using the $3 \mathrm{D}$ printing technique with polyamide PA12. To obtain equal powers and symmetry of diffracted beams, the computer-aided optimization algorithm was used. Diffractive optical elements designed for 140 and $282 \mathrm{GHz}$ were evaluated experimentally at both these frequencies using illumination with the wavefront coming from the point-like source. Described fan-out elements formed uniform intensity and equidistant energy distribution in agreement with the numerical simulations.
\end{abstract}

Keywords Terahertz $\cdot$ Beam splitting $\cdot 3$ D printing $\cdot$ Diffractivelens $\cdot$ Dammann grating $\cdot$ Fan-out elements

\section{Introduction}

The aim of this study was to design, manufacture, and examine diffractive optical structures that divide the light into a precise number of beams in the THz range. They can be used for fan-out applications, for example for $\mathrm{THz}$ communication [1] and space observation [2]. Moreover, they can be applied in cases where it can be helpful to illuminate the object with a larger number of points (or lines) to increase the spatial resolution of scanning or imaging. For example, the THz postal scanner [3] that utilizes a hyperbolic-lens-like structure to focus

K. Liebert

Karolina.liebert@gmail.com

1 Faculty of Physics, Warsaw University of Technology, Warsaw, Poland

2 Charles Coulomb Laboratory (L2C) - UMR5221 CNRS, University Montpellier, Montpellier, France 
the beam from the source into a one line segment in the focal plane was previously described. Such a line-shape wavefront propagates through the scanned medium and it is then registered by the field effect transistors (FET) [4]. The FET detectors cannot be placed close to each other due to the soldering difficulties. Placing them in the three-line-configuration, where each is shifted in relation to the other, reduces the problem of low resolution. For the three lines of detectors to work correctly, it was necessary to split the illumination beam into three. As a result of that arrangement, the spatial resolution can be significantly increased [5]. Multiple light beams with similar power distribution are also crucial in guiding and positioning systems [6]. The fan-out systems that operate in the $\mathrm{THz}$ range can also be successfully applied on board of helicopters to improve safety while encountering the brown-out or white-out when landing [7].

Nowadays, commercial fan-out elements for visible light split the incident light into a defined number of diffraction orders with equal powers. Moreover, the advanced shaping of the beam (its controlling and changing into different patterns) can be most effectively realized by diffractive optical elements [8]. Such elements are widely used for contact-less testing, metrology, remote sensing etc. In case of fan-out elements an array of dots (or different shapes like lines, circles, etc.) can be generated by the use of either Dammann gratings [9] or periodic structures coded as kinoforms (continuous-relief elements) [10]. They can obtain diffraction efficiencies up to 80 and $95 \%$, respectively [11]. By using more complicated structures, we can obtain fan-out optical elements (OE) optimized with Gerchberg-Saxton algorithm [12] or OE that generate sub-diffraction-limit spot arrays [13]. The continuous-relief elements are difficult to manufacture and are sensitive to fabrication errors. That can significantly decrease the uniformity of the generated diffraction orders intensity [14]. Although the fan-out elements based on Dammann gratings have lower diffractive efficiencies, they are much simpler in manufacturing. Furthermore, their optimization algorithms are already well-known [9].

Using fan-out elements for the THz range of radiation seems a very desiring and interesting solution, therefore, we demonstrated two types of such structures: (i) the $3 \times 1$ matrix fan-out structure based on the optimized binary phase grating and (ii) the $3 \times 3$ fan-out structure designed on the basis of the well-known Dammann grating $[9,15,16]$. Nevertheless, it should be underlined that the Dammann grating used in the visible range of electromagnetic waves consists of thousands of periods. For the wavelength in the range of millimeters, only few periods form the whole structure. Therefore, the optimization process is necessary [17]. Originally, the Dammann grating is a binary phase $(0, \pi)$ structure forming an array illumination. Each period of such a grating consists of rectangular elements introducing either 0 or $\pi$ phase retardation. In case of binary phase transmittances, we can expand the theoretical 1D considerations into a $2 \mathrm{D}$ grating by multiplying two $1 \mathrm{D}$ optical elements.

Most of the fan-out structures are designed for an ideal plane wave illumination. In this case, there is a need for two optical elements: one to form a plane wave and a second one to form the fan-out motif. The structures proposed in this article were designed to be illuminated directly with the divergent wavefront coming from $\mathrm{THz}$ source (e.g., directly from the horn). The modeled structures consisted of the described optimized phase grating and a converging lens. As a result, 2-in-1 structures were obtained that can replace two independent elements: a focusing lens and a separating optical fan-out element.

Due to our broad experience in designing $\mathrm{THz}$ optical elements (broad band double side lenses [18], high-order kinoforms [19], diffractive paper lenses [20], hyperbolical lenses [3], lens-like structures with different focal curves [21, 22], different materials can be listed as possible solutions of manufacturing described fan-out elements: paper [20], high-density 
polyamide - HDPE [18], polyamide 12 - PA12 [3], metal [23] etc. Moreover, depending on used material various manufacturing techniques must be used, like 3D printing [24], milling, laser cutting, etc. The 3D printing technology is fast growing field [25-27]. The structures proposed in this paper were created in the selective laser sintering 3D printing technology (SLS), which allows good enough resolution. PA12 material was chosen for structure production for its relatively good transmission, and possibility of printing from it on SLS [28, 29]. Manufactured structures were lightweight, durable, and low-cost that is indispensable in outdoor applications, or in other adverse conditions. For predetermined wavelengths (1.064 and $2.141 \mathrm{~mm}$, corresponding to 140 and $282 \mathrm{GHz}$ ) manufacturing accuracy was sufficient and fulfilled the optical requirements.

\section{Design of Fan-Out Element Forming $3 \times 1$ and $3 \times 3$ Arrays}

The numerical modeling for the $3 \times 1$ fan-out structure is based on a basic binary grating with $0-\pi$ phase retardation. This type of the structure has never been created for the THz range of frequencies. The $3 \times 1$ fan-out structure was designed and manufactured for two particular types of sources that generate the radiation with frequencies 140 and $282 \mathrm{GHz}$. For the $3 \times 1$ element, the algorithm used to optimize parameters of the binary phase grating was developed assuming finite dimensions of the structure. We analyzed different values of $\varphi$-phase delay introduced by the grating and different values of its shift $-S$ (the grating can be ideally symmetrical or not), marked in Fig. 1.

As a result of the optimization algorithm (Fig. 2), two parameters: $\varphi_{\text {OPTIMAL }}$ and $S_{\text {OPTIMAL }}$ were determined for a specific wavelength $\lambda$ and an angle of diffraction (closely related to the grating period).

At first, the optimization of the grating shift- $S$ was carried out to obtain perfectly symmetrical output beams in \pm 1 order of diffraction. The initial phase retardation was set to $\pi$. As a result a phase grating with a 0.5 fill factor was obtained. Such a grating deflects in summary $81 \%$ of the incoming radiation on +1 st and -1 st orders (and the rest of the energy is redirected into higher odd orders of diffraction). To focus some energy into 0th order of diffraction, it was necessary to change the introduced phase delay (from $\pi$ value). The phase delay value was optimized until equal intensity in +1 st, -1 st and 0 th orders of diffraction was obtained. At the end of the algorithm, the phase distribution of the converging lens was added to the phase distribution of the grating. Introducing the lens with focal length equal to $300 \mathrm{~mm}$ corrected the divergent wavefront coming from the point-like source placed at a distance of $Z_{A}=300 \mathrm{~mm}$ before the fan-out structure.

Fig. 1 The schematic of the diffraction grating phase profile. Optimized parameters $\varphi$ (phase step value) and $S$ (shift of the grating) are marked. This graph corresponds to the cross-section of the designed structure, where $X$ is its horizontal axis and $Y$ is the height of the structure (strictly referred to its phase retardation)

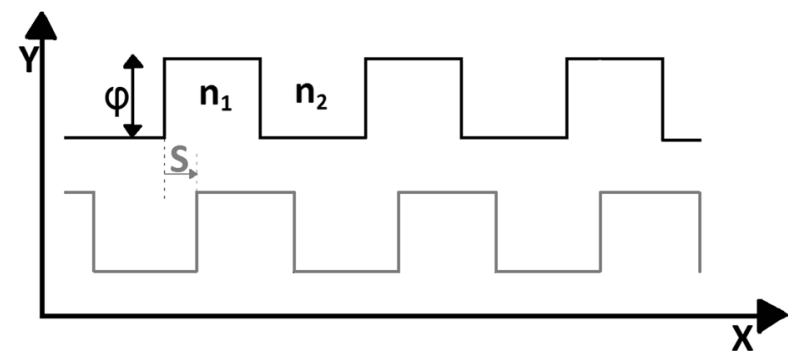


Fig. 2 The block diagram of the algorithm of optimizing the $3 \times 1$ fan-out element. The initial phase value $\varphi_{0}$ is equal to $\pi$ and $S$ is equal to 0 . The first propagation at a distance $Z_{A}=300 \mathrm{~mm}$ and $Z_{A}=495 \mathrm{~mm}$ distance which was also the focal length of the compensating converging lens. The intensity distribution (modulus square of the measured amplitude) was modeled for three different distances: $Z_{B}=100 \mathrm{~mm}$, $Z_{B}=300 \mathrm{~mm}, Z_{B}=900 \mathrm{~mm}$

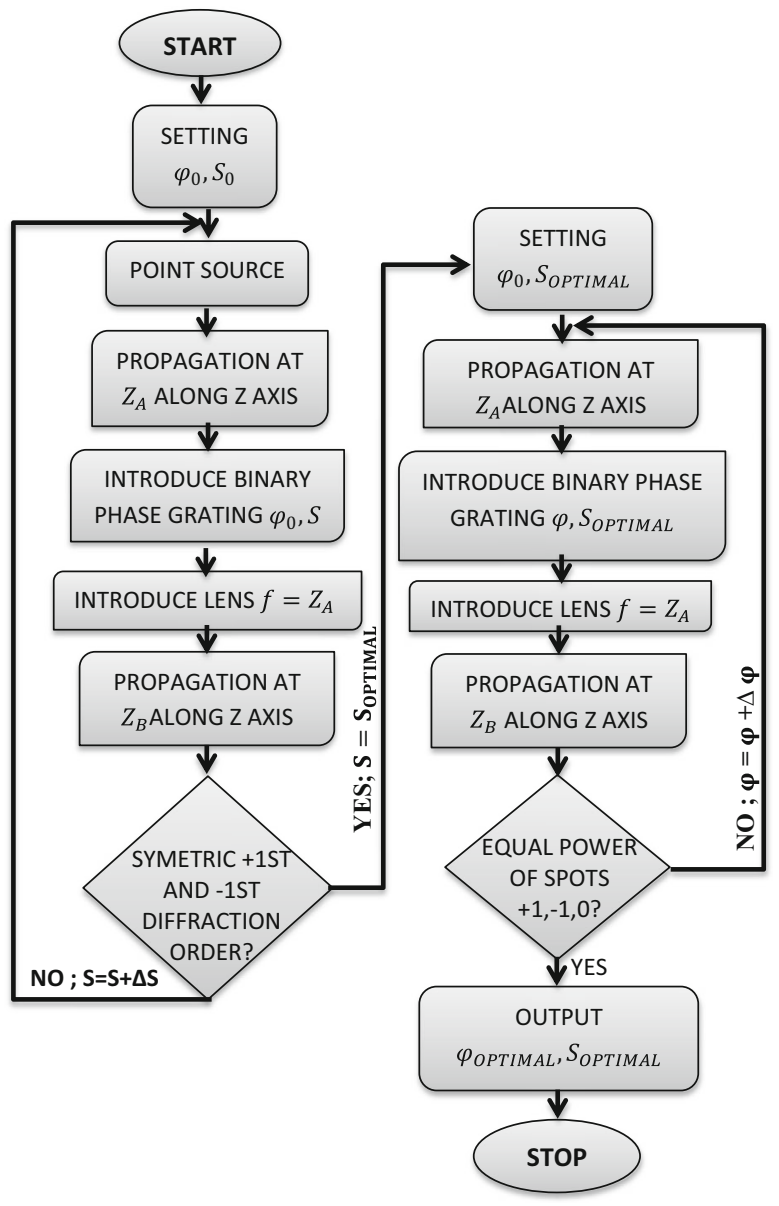

The $3 \times 3$ fan-out structure design was carried out on the basis of the Dammann gratings that are described in the literature [9, 15-17]. The designed fan-out element consisted of the Dammann grating that is able to create a $3 \times 3$ array of points with the diffraction angle of $6^{\circ}$ and a converging lens (with a focal length $495 \mathrm{~mm}$ ). Such focal length compensates the diverging wavefront coming from the point-like source located at a distance $Z_{A}=495 \mathrm{~mm}$ before the designed element. This element was designed only for the wavelength equal to $1.064 \mathrm{~mm}$ corresponding to the frequency of $282 \mathrm{GHz}$.

\section{Numerical Modeling Results}

The modeling of all elements was carried out on calculation arrays of $4096 \times 4096$ pixels with the sampling of $117 \mu \mathrm{m} \times 117 \mu \mathrm{m}$ for both frequencies: 140 and $282 \mathrm{GHz}$. Sampling used in the simulations was determined by the precision of the manufacturing device. The structures were modeled on square calculation arrays with the assumption that they are illuminated by the point-like source. The real size of the horn was also considered in the computer modeling. 
Figure $3 \mathrm{a}$ presents the phase distribution of the $3 \times 1$ fan-out $\mathrm{OE}$ designed for $140 \mathrm{GHz}$ (design wavelength $\mathrm{DWL}=2.141 \mathrm{~mm}$ ) with the grating period of $21.57 \mathrm{~mm}$ for the diffraction angle of about $6^{\circ}$. Figure $3 \mathrm{~b}$ shows the phase distribution of the $3 \times 1$ fan-out $\mathrm{OE}$ for $282 \mathrm{GHz}$ (DWL $1.064 \mathrm{~mm}$ ) with the grating period equal to $10.7 \mathrm{~mm}$ for the same diffraction angle.

The scheme of the modeled systems is shown in Fig. 4. The numerical modeling was performed with the use of LightSword 6.0 software (LS 6.0) which is based on the wave approach of the propagation phenomenon modeling. Propagation in free space is calculated on the basis of the decomposition of complex light waves into plane waves. Additionally, for better consistence with experimental results, the volume algorithm based on modified beam propagation method (mBPM) [30] was used. Source spatial dimensions were modeled to correspond with dimensions of experimental setups. We assumed the source diameter of 18 and $6.5 \mathrm{~mm}$, respectively for 140 and $282 \mathrm{GHz}$.

The intensity distribution was examined for three different distances $\left(Z_{B}\right): 100,300$, and $900 \mathrm{~mm}$. The computer modeling results are presented in Tables 1 and 2 in Section 5 .

For the $3 \times 3$ fan-out element case, the structure was designed only for the frequency of $282 \mathrm{GHz}$ (DWL $1.064 \mathrm{~mm}$ ). The $3 \times 3$ fan-out optical element consisted of the Dammam grating with $10.7-\mathrm{mm}$ period and a focusing lens with the focal length of $495 \mathrm{~mm}$. The structure was designed as a circle with a diameter of $160 \mathrm{~mm}$. Because of bigger diameter of the structure the distance from the source to the structure was enlarged to assure uniform illumination of the OE. Figure 5a presents the phase distribution of the designed $3 \times 3$ fan-out OE for $282 \mathrm{GHz}$ and Fig. $5 \mathrm{~b}$ the scheme of the modeled system. Due to the experimental setup configuration, the designed $\mathrm{OE}$ was illuminated with the divergent wavefront (radiating from the point-like source distant $Z_{A}=495 \mathrm{~mm}$ from it). The separation of diffracted beams occurred relatively far away and could not have been easily measured by the used detector. The intensity pattern in the far-field diffraction region (Fraunhofer diffraction zone) is described by the Fourier transform of the input wavefront (with scaling factor). Therefore, we decided to perform the measurement of the corresponding intensity pattern in the Fourier plane of the additional lens. The intensity pattern observed in this plane is qualitatively the same as the pattern in the far-field region. Thus, an additional converging lens with focal length $f=300 \mathrm{~mm}$ and diameter $d=300 \mathrm{~mm}$ was added. It focused the radiation and the distribution was scanned in its Fourier plane.

a)

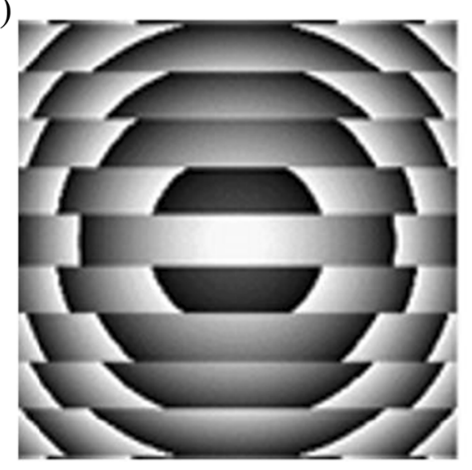

b)

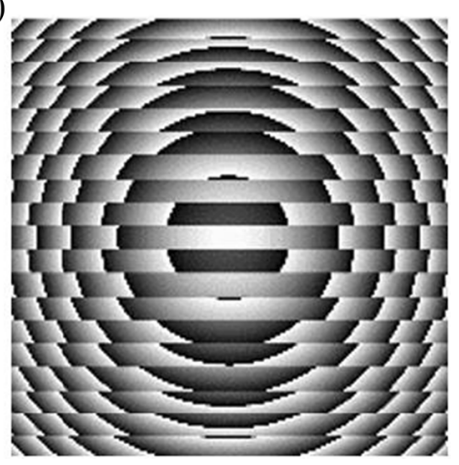

Fig. 3 The phase distribution of the $3 \times 1$ fan-out optical element for a 140 and b $282 \mathrm{GHz}$ 
Fig. 4 The scheme of the modeling setup for the $3 \times 1$ optical elements, for both frequencies, 140 and $282 \mathrm{GHz}$

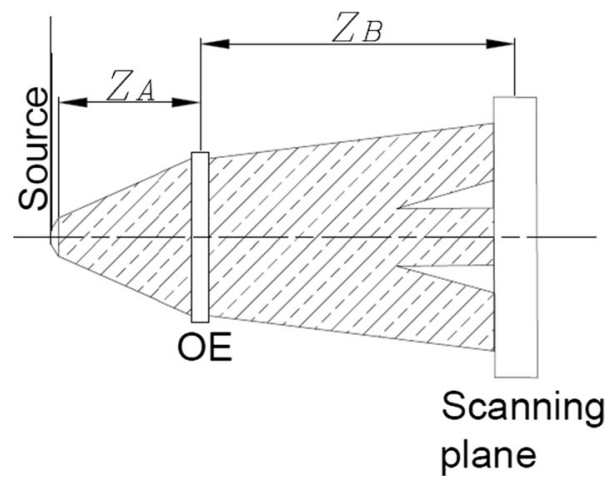

The intensity distribution was modeled for the distance $Z_{B}=300 \mathrm{~mm}$ behind the additional converging lens (in Fourier plane). The computer modeling results are presented in Fig. 9 (results Section 5).

\section{Experimental Results}

All three optical elements were manufactured using the laser sintering 3D printing method [18] with polyamide 12 (PA12) material. The refractive index of PA12 material for both frequencies was $n=1.59$. These parameters were determined using the TDS system prior to the start of the experiment.

Created structures had spatially variable thickness (they were kinoform structures and their photographs are shown in Fig. 6), therefore calculation of exact attenuation of the elements was very difficult. For $140 \mathrm{GHz}$ the structure thickness varied from $1 \mathrm{~mm}$ (thickness of the substrate layer) to $4.6 \mathrm{~mm}$ (highest phase shift area) with absorption coefficient equal to $0.3 \mathrm{~cm}^{-1}$, the attenuation would change from 0.03 to $0.14 \mathrm{~dB}$. The thickness of the structure for $282 \mathrm{GHz}$ varied from 1 to $2.8 \mathrm{~mm}$ with absorption coefficient equal to $1.6 \mathrm{~cm}^{-1}$, so the absorption should change in between $0.16-0.45 \mathrm{~dB}$.

Table 1 The comparison of the computer modeling and the experimental results for the $3 \times 1$ fan-out OE for $140 \mathrm{GHz}$ frequency at the distances $Z_{B}$ equal to 100,300 , and $900 \mathrm{~mm}$

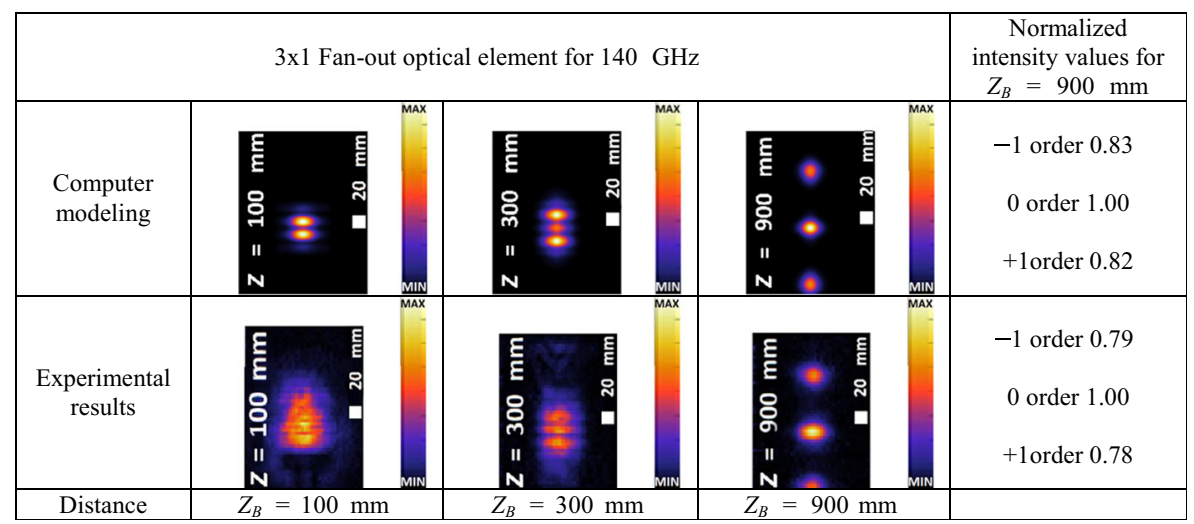


Table 2 The comparison of the computer modeling and the experimental results for the $3 \times 1$ fan-out OE for $282 \mathrm{GHz}$ frequency at the distances of 100,300 , and $900 \mathrm{~mm}$

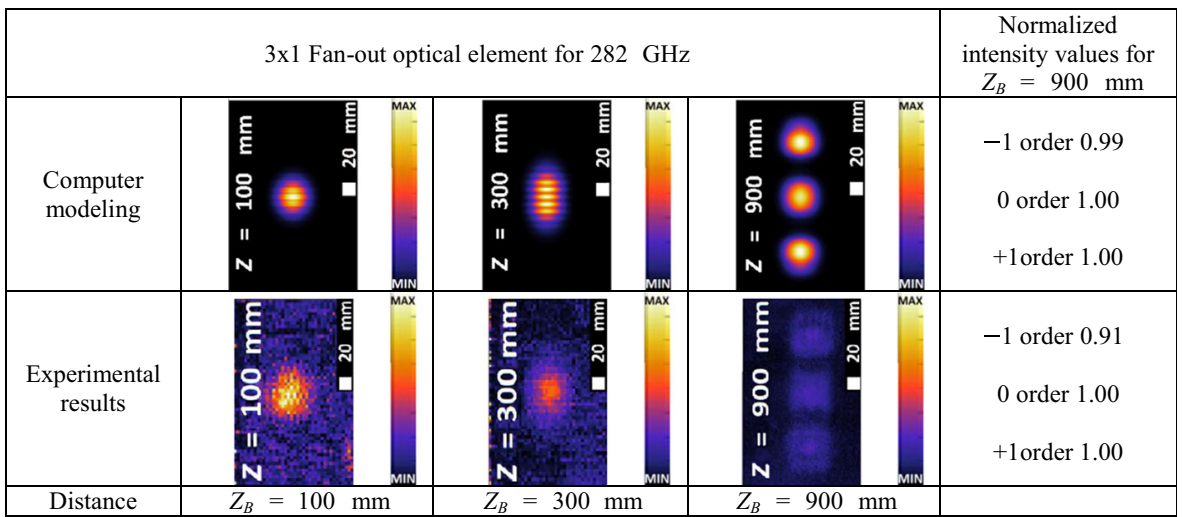

The source used in the optical system had power of $29 \mathrm{~mW}$ for $140 \mathrm{GHz}$ and $4.5 \mathrm{~mW}$ for $282 \mathrm{GHz}$. The pyroelectric sensor used as a detector for both wavelengths had the sensitivity of $44.8 \mathrm{~V} / \mathrm{W}$. Moreover, the diameter of pyroelectric window was $20 \mathrm{~mm}$. However, the scanning step was equal to $5 \mathrm{~mm}$ to increase the resolution, which is visible in experimental results (Tables 1 and 2 and Fig. 9).

The scheme of the optical system used to measure the $3 \times 1$ fan-out element for 140 and $282 \mathrm{GHz}$ frequencies is shown in Fig. 7. Additionally, to increase the sensitivity of the optical system (emitter-detector) the lock-in system was used.

The experimental results showing the intensity distribution were examined for three different distances $\left(Z_{B}\right) 100,300$, and $900 \mathrm{~mm}$ and are presented in Table 1 for $140 \mathrm{GHz}$ and Table 2 for $282 \mathrm{GHz}$ in Section 5.

The scheme of the experimental optical system for the $3 \times 3$ fan-out element for $282 \mathrm{GHz}$ case is shown in Fig. 8a. The additional converging lens was used here (shown in Fig. 8b) to focus the radiation into Fourier plane instead of Fraunhofer diffraction region. This lens was HDPE lens that was previously designed and was a high order kinoform [19] having the focal length equal to $300 \mathrm{~mm}$ and the diameter equal to $300 \mathrm{~mm}$. Thus, the Fourier plane was located at a distance of $300 \mathrm{~mm}$ behind the converging lens (where the scanning plane was). The manufactured $3 \times 3$ fan-out optical element is presented in Fig. $8 \mathrm{c}$.

a)

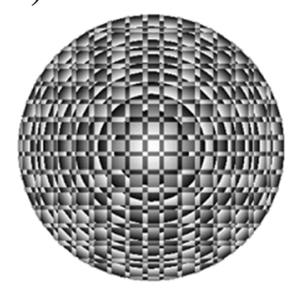

b)

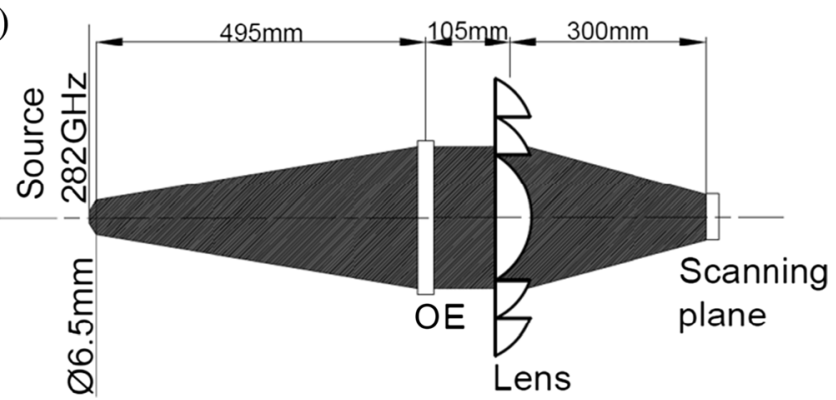

Fig. 5 a The phase distribution of the $3 \times 3$ fan-out optical element designed for $282 \mathrm{GHz}$, b the scheme of the modeled optical system for this frequency 
a)

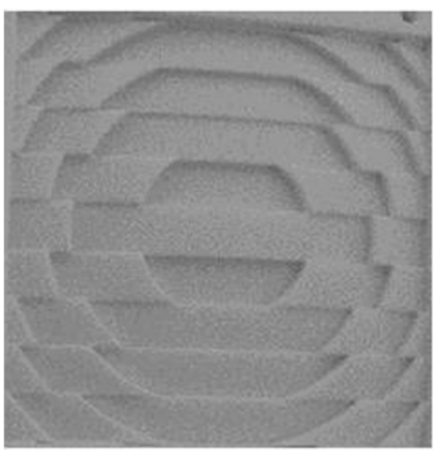

b)

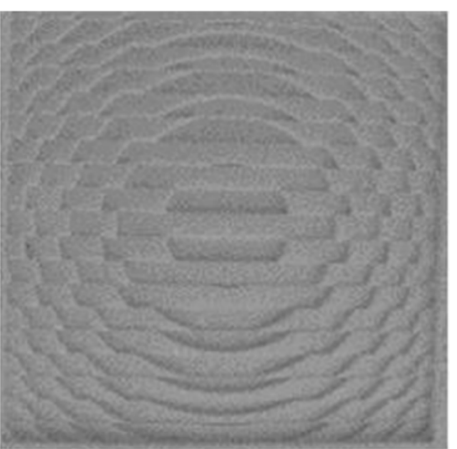

Fig. 6 The manufactured $3 \times 1$ fan-out optical elements with the side length equal to $100 \mathrm{~mm}$ for a 140 and $\mathbf{b}$ $282 \mathrm{GHz}$

Originally, the designed and manufactured structure for the $3 \times 3$ fan-out element case originally had a diameter of $160 \mathrm{~mm}$. To obtain a satisfactory separation of diffracted beams at a distance $Z_{B}=300 \mathrm{~mm}$, the optical element was further limited with the metallic aperture absorbing THz radiation attached to the flat side of the lens. This aperture limited the active diameter to $80 \mathrm{~mm}$. The photograph of the structure with the marked size of the aperture is presented in Fig. 8c. In the experimental evaluation, we decided to limit the aperture because then we obtained better results which could be due to the fact that the compensating lens was designed in paraxial approach. Therefore, it could introduce mistakes at the edges of the structure causing its lower efficiency, and the aperture was finally limited. Additionally, shadow effect could decrease the efficiency at the edges [31]. The experimental results showing the intensity distribution were examined for the distance of $300 \mathrm{~mm}$ and are presented in Fig. 9 in Section 5.

\section{Results}

As previously mentioned, the pyroelectric window had the diameter equal to $20 \mathrm{~mm}$. The sampling in computer simulations was $117 \mu \mathrm{m} \times 117 \mu \mathrm{m}$ (resulting from production process requirements and accuracy). Therefore, in numerical modeling process the size of the detector was taken into account (as a convolution of the simulation data with the aperture of detector

Fig. 7 The scheme of the experimental optical system for the $3 \times 1$ OE. $140 \mathrm{GHz}$ source diameter was $18 \mathrm{~mm}$ and $282 \mathrm{GHz}$ source diameter was equal to $6.5 \mathrm{~mm}$

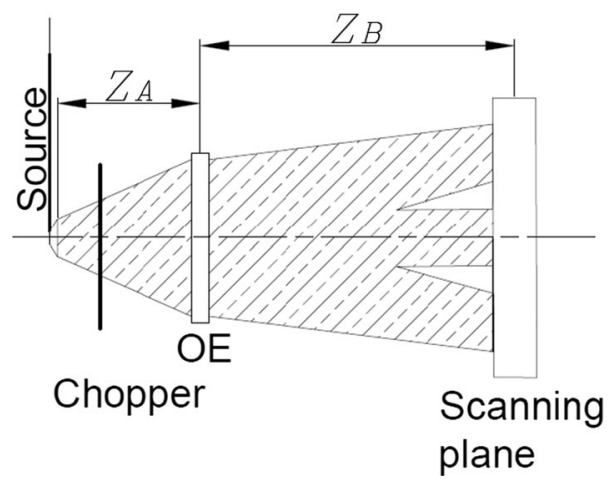


a)

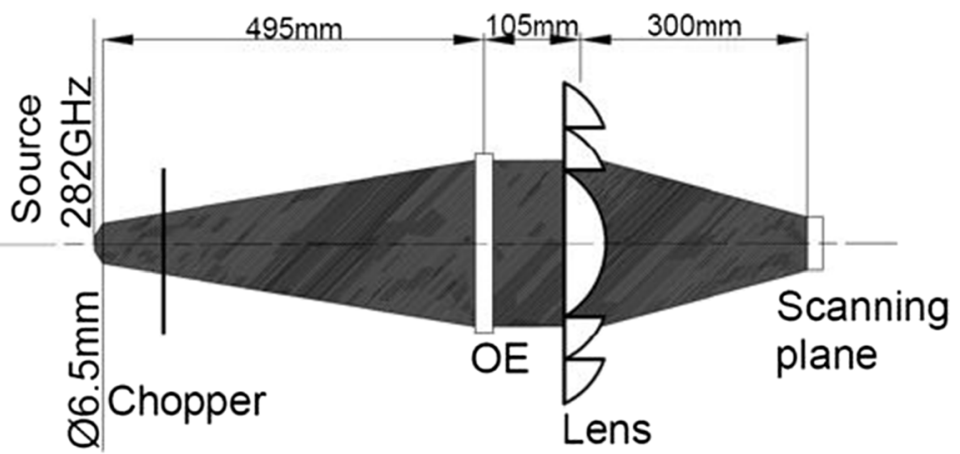

b)

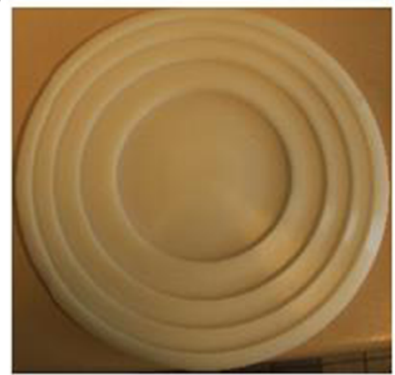

c)

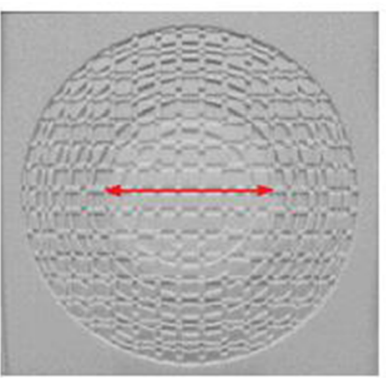

Fig. 8 The $3 \times 3$ fan-out element case, for $282 \mathrm{GHz}$. a The scheme of the real optical system, b the additional converging lens used in the optical system having $f=300 \mathrm{~mm}$ and $d=300 \mathrm{~mm}$ made from HDPE material, which was used to shorten the necessary measurement distance. $\mathbf{c}$ The manufactured $3 \times 3$ fan-out optical element with a side length equal to $160 \mathrm{~mm}$. The red arrow indicated limited area to the diameter of $80 \mathrm{~mm}$

window) that enabled a comparison between results obtained for the computer modeling and the experiment.

Table 1 presents the modeling and the experimental results for the $3 \times 1$ fan-out optical element for $140 \mathrm{GHz}$.

At the distance $Z_{B}=900 \mathrm{~mm}$ there were exactly three separated points with similar intensities. The element was designed as an optimized diffraction grating which theoretically forms the desired intensity pattern in the infinity. In the experimental setup, the measurement of the intensity pattern was carried out at a distance corresponding to the far-field region

a)

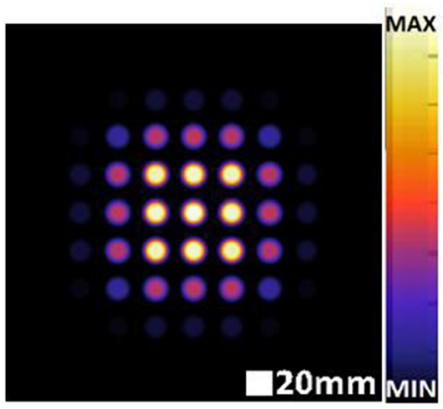

b)

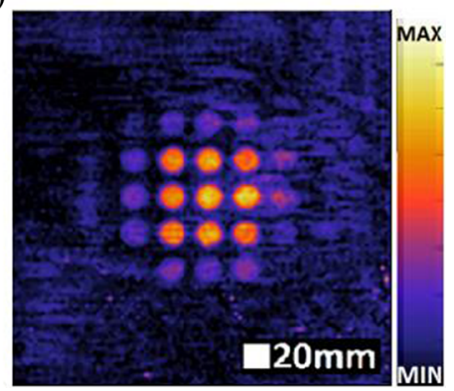

Fig. 9 The comparison of the computer modeling and the experimental data for the $3 \times 3$ fan-out element case for $282 \mathrm{GHz}$ frequency. In the modeling process the size of the detector was taken into account 
$\left(Z_{B}=900 \mathrm{~mm}\right)$. For the designed diameter of the structure, it can be assumed that the distance of $900 \mathrm{~mm}$ belongs to the far-field region. For the THz frequencies, the OE consisted of a small number of periods which additionally reduced its efficiency and led to the nonuniformity of intensity in all three diffraction orders.

The intensity of the 0th order spot was bigger (around 20\%), but it should be underlined that all intensities should be equal in infinity. As it is shown in intensity levels, theoretical and experimental intensity distribution variation is similar. The results of the intensity distribution were normalized to 0th order of diffraction to clearly present the uniformity of intensity distribution between the three beams. It is worth noticing that the +1 st and -1 st orders of diffraction were almost equal, therefore the algorithm optimizing symmetry was carried out properly.

The source of $282 \mathrm{GHz}$ radiation had smaller power, which resulted in a relatively large noise to information ratio in the experimental results. Additionally, although the structure for $282 \mathrm{GHz}$ was thinner, it transmitted less radiation than structure designed for $140 \mathrm{GHz}$. It was a result of PA12's bigger attenuation coefficient value for higher frequency.

While discussing the results illustrated in Table 2, it should be taken into consideration that the frequency of $282 \mathrm{GHz}$ was likely to be the limit of the sensitivity of the detector. Nevertheless, the three spots were separated properly corresponding to the simulation results but they had less energy in comparison with the results obtained for $140 \mathrm{GHz}$. For both frequencies, 140 and $282 \mathrm{GHz}$, we were able to optimize the structure, so that three spots, that had similar intensities were visible in the far-field region (here $Z_{B}=900 \mathrm{~mm}$ ). As it can be seen, the size of the spots in Table 2 is bigger than in Table 1. It corresponds to the theory stating that for the same aperture size of the element the spot size depends on the frequency (directly proportional).

Figure 9 illustrates the intensity distribution of the computer modeling and the experimental data in the Fourier plane. The separation of spots in a $3 \times 3$ array is consistent with simulation predictions and the intensities of spots are similar. The additional beams that are quite well seen in the simulation results are almost not seen in the experiment due to their value being similar to the registered noise.

\section{Conclusion}

Depending on the final use of the fan-out elements, a pre-defined number of diffracted beams can be easily formed. Diffraction efficiency of the presented optical elements is high enough to provide a full separation at a particular distance (corresponding to the Fraunhofer region). One durable structure can replace the need to use a matrix of sources. It radically simplifies the design and reduces the mechanical sensitivity of the system. It will also enable to preserve mutual coherence of multiple beams in case when it is needed.

In the experiment, the $3 \times 3$ fan-out structure showed better efficiency and therefore better results. That is caused mostly by the fact that additional converging lens was used in the experiment, which enabled observation not in the far-field region but in the Fourier plane and allowed to obtain larger intensities of spots in array. Moreover, in comparison with the $3 \times 1$ fan-out structure, the $3 \times 3$ fan-out structure was a Dammann-like structure that was optimized to assure uniform distribution between all the spots in the designed array. 
Therefore, the designed $3 \times 1$ and $3 \times 3$ fan-out structures may have the practical use in illuminating systems in the $\mathrm{THz}$ range of radiation including the $\mathrm{THz}$ scanners [3], the $\mathrm{THz}$ multipoint communication systems [1], and the space observation instruments [2].

Acknowledgements The authors would like to thank Orteh Company for providing LightSword 6.0 (LS 6.0) Software for designing and modeling diffractive optical elements and also for manufacturing investigated structures.

This work was partially supported by the Laboratoire International Associé "TERAMIR" and National Science Centre (Poland), allocated on the basis of decision number DEC-2013/10/M/ST3/ 00705 .

Open Access This article is distributed under the terms of the Creative Commons Attribution 4.0 International License (http://creativecommons.org/licenses/by/4.0/), which permits unrestricted use, distribution, and reproduction in any medium, provided you give appropriate credit to the original author(s) and the source, provide a link to the Creative Commons license, and indicate if changes were made.

\section{References}

1. M. N. Armenise, M. Armenise, R. Diana, Novel integrated optical beam former for phased-array antennas, Proc. SPIE3464, Optical Devices and Methods for Microwave/Millimeter-Wave and Frontier Applications, 95 (October 2, 1998)

2. J. Leech, S. Withington, G Yassin, An SIS focal plane imaging array for $350 \mathrm{GHz}$, IEEE. High Frequency Postgraduate Student Colloquium, 1999. 146-151 (1999)

3. J. Suszek, A. Siemion, M. S Bieda, N. Blocki, D. Coquillat, G. Cywinski, E. Czerwinska, M. Doch, A. Kowalczyk, N. Palka, A. Sobczyk, P. Zagrajek, M. Zaremba, A. Kolodziejczyk, W. Knap, M. Sypek, 3-Dprinted flat optics for THz linear scanners, IEEE Transactions on Terahertz Science and Technology 5(2) 13 (2015)

4. W. Knap, M. Dyakonov, D. Coquillat et al. Field effect transistors for terahertz detection: physics and first imaging applications. J. Infrared Millim. Te. 30, 1319 (2009)

5. http://orteh.pl/eng/articles/show/22 (accessed 2017 May 4)

6. I. Moir, A. Seabridge, and M. Jukes, Military avionics systems, John Wiley Sons Inc, 2013

7. E. Rash "Flying Blind", Flight Safety Foundation, Aviation Safety World, 44-46 (2006)

8. http://holoeye.com/diffractive-optics/ (accessed 2017 May 4)

9. H. Dammann and K. Gortler, High-efficiency in-line multiple imaging by means of multiple phase holograms, Opt. Commun. 3, 312-315 (1971)

10. H. P. Herzig, D. Prongué, and R. Dändliker. Design and fabrication of highly efficient fan-out elements. Japanese journal of applied physics 29.7A (1990)

11. H. P. Herzig. Micro-Optics: Elements, Systems And Applications. Institute of Microtechnology, University of Neuchatel, Switzerland, CRC Press, 16-21 (1997)

12. Y. Ogura, M. Aino, and J. Tanida, Design and demonstration of fan-out elements generating an array of subdiffraction spots, Opt. Express 22, 25196-25207 (2014)

13. Y. Ogura, M. Aino, and J. Tanida, Diffractive fan-out elements for wavelength-multiplexing subdiffractionlimit spot generation in three dimensions, Appl. Opt. 55, 6371-6380 (2016)

14. J. Bengtsson and M. Johansson, Fan-out diffractive optical elements designed for increased fabrication tolerances to linear relief depth errors, Appl. Opt. 41, 281-289 (2002)

15. H. Dammann and E. Klotz, Coherent optical generation and inspection of two-dimensional periodic structures, Opt. Acta. 24, 505-515 (1977)

16. M. Goel, D. L. Naylor, Analysis of design strategies for Dammann gratings, Proc. SPIE 2689, Diffractive and Holographic Optics Technology III, 35 (1996)

17. C. Zhou and L. Liu, Numerical study of Dammann array illuminators, Appl. Opt. 34, 5961-5969 (1995)

18. M. Sypek, M. Makowski, E. Hérault, A. Siemion, A. Siemion, J. Suszek, F. Garet, and J.-L. Coutaz, Highly efficient broadband double-sided Fresnel lens for THz range, Opt. Lett. 37, 2214-2216 (2012)

19. J. Suszek, A. M. Siemion, N. Błocki, M. Makowski, A. Czerwiński, J. Bomba, A. Kowalczyk, I. Ducin, K. Kakarenko, N. Pałka, P. Zagrajek, M. Kowalski, E. Czerwińska, C. Jastrzebski, K. Świtkowski, J.-L. Coutaz, A. Kolodziejczyk, and M. Sypek, High order kinoforms as a broadband achromatic diffractive optics for terahertz beams, Opt. Express 22, 3137-3144 (2014) 
20. A. Siemion, A. Siemion, M. Makowski, J. Suszek, J. Bomba, A. Czerwiński, F. Garet, J.-L. Coutaz, and M. Sypek, Diffractive paper lens for terahertz optics, Opt. Lett. 37, 4320-4322 (2012)

21. A. Siemion, J. Suszek, A. Kowalczyk, J. Bomba, A. Sobczyk, N. Palka, P. Zagrajek, A. Kolodziejczyk, M. Sypek, THz beam shaping based on paper diffractive optics, IEEE Transactions on Terahertz Science and Technology, 6 (4) 568-575 (2016)

22. A. Siemion, P. Kostrowiecki-Lopata, A. Pindur, P. Zagrajek, and M. Sypek, Paper on designing costless THz paper optics, Advances in Materials Science and Engineering, 2016 (2016)

23. A. Siemion, A. Siemion, M. Makowski, M. Sypek, E. Hérault, F. Garet, J.L. Coutaz, Off-axis metallic diffractive lens for terahertz beams. Optics letters, 36(11), 1960-1962 (2011)

24. T. N. A. Tuan Rahim, A. M. Abdullah, H. Md Akil, et al., Preparation and characterization of a newly developed polyamide composite utilizing an affordable 3D printer. Journal of Reinforced Plastics and Composites, 34(19): 1628-1638 (2015)

25. S. F. Busch, M. Weidenbach, J. C. Balzer and M. Koch, THz optics 3D printed with TOPASJ. Infrared Millim. Te 37(4), 303-307 (2015)

26. D. Jahn, M. Weidenbach, J. Lehr, 3D Printed Terahertz Focusing Grating Couplers. J Infrared Millim. Te 1-9 (2017)

27. A. D. Squires, E. Constable and R. A. Lewis, 3D Printed Terahertz Diffraction Gratings And Lenses. J Infrared Millim. Te 36(1), 72-80 (2015)

28. K. Szkudlarek, M. Sypek, G. Cywiński, et al. Terahertz 3D printed diffractive lens matrices for field-effect transistor detector focal plane arrays, Opt. Express 24, 20119-20131 (2016)

29. S. F. Busch, M. Weidenbach, M. Fey, et al., Optical Properties of 3D Printable Plastics in the THz regime and their Application for 3D Printed THz Optics J Infrared Millim. Te 35(12), 993-997 (2014)

30. M. Sypek, Light propagation in the Fresnel Region. New numerical approach, Opt. Commun., 116, 43-48 (1995)

31. J. Suszek, M. Sypek, M. Makowski, F. Garet, I. Ducin, K. Kakarenko, J. Bomba, and J.-L. Coutaz, Evaluation of the shadow effect in terahertz kinoform gratings, Optics Letters 38, no. 9, 1464-1466 (2013) 